\title{
MAGNETIC PITCH ANGLE MEASUREMENTS \\ ON TEXT USING LASER-ENHANCED ATTENUATION OF A Li BEAM
}

by

W.P. WEST, J. McCHESNEY, M.K. THOMAS, and X.Z. YANG*

This is a preprint of a paper to be presented at the Eighth Topical Conference on High-Temperature Plasma Diagnostics, May 7-10, 1990, in Hyannis, Massachusetts and to be printed in the Proceedings.

\author{
Work supported by \\ U.S. Department of Energy \\ Contract DE-AC03-89ER53277
}

*Permanent address: Chinese Academy of Scierices, Beijing, PRC.

GENERAL ATOMICS PROJECT 3468

SEPTEMBER 1990

w

6.t.

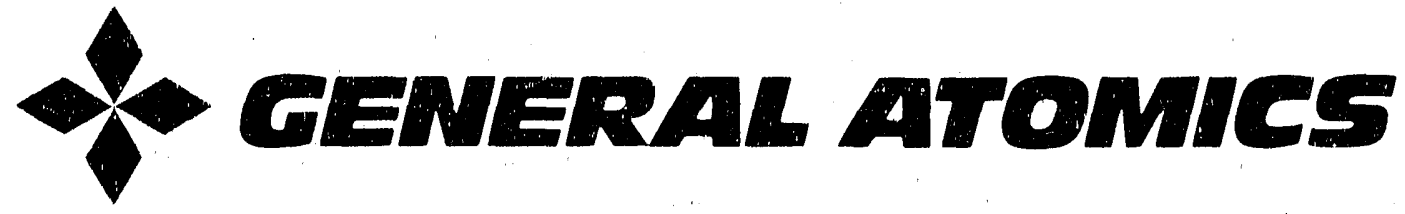




\title{
Magnetic pitch angle measurements on TEXT using laser-enhanced attenuation of a $\mathrm{Li}$ beam
}

W.P. West, J. McChesney, M.K. Thomas, and X.Z. Yang*

General Atomics, San Diego, California 92186-9084

* Fusion Research Center, University of Texas at Austin

Permanent address, Chinese Academy of Sciences, Beijing, PRC

\begin{abstract}
The polarization of the $\pi$ component of the Zeeman split Li2P-2S transition allows a direct spectroscopic measurement of the local magnetic pitch angle. Li atoms are injected radially into TEXT as a $95 \mathrm{keV}$ beam, and the intensity of the transmitted beam is detected. A polariration-modulated, cavity-dumped dye laser, tuned intr, resonance with the $\pi$ line, is injected collinearly with the beam. Laser excitation of the beam results in an enhanced beam attenuation. Time-resolved detection of the transmitted $\mathrm{Li}$ beam allows determination of the local magnetic pitch angle. Details of the system and results of recent measurements will be discussed.
\end{abstract}




\section{INTRODUCTION}

An adequate current profile diagnostic system suitable for the wide variety of tokamaks in the community remains elusive. While many techniques have been demonstrated to work under limited conditions (too many to reference in this brief paper), a generally applicable diagnostic tool has not yet been demonstrated. We are developing a system for the TEXT tokamak that promises to be applicable to most tokamaks of similar or smaller size and promises to obtain complete profiles with reasonable time resolution. This system design stems from previous work on TEXT, using a $\mathrm{Li}$ beam and laserinduced fluorescence (LIF) to measure magnetic pitch angle profiles. ${ }^{1,2}$ During the course of the previous work, it was discovered that laser excitation of the $\mathrm{Li}$ beam atoms led to an enhancement of the $\mathrm{Li}$ beam attenuation, resulting in reduced fluorescence and limiting the density range of applicability to $<1 \times 10^{13} \mathrm{~cm}^{-3}$. The present system takes advantage of laser-enhanced attenuation (LEA) to measure magnetic pitch angle profiles.

An injected atomic Li beam allows magnetic pitch angle measurements because of the resonance line splitting and polarization resulting from the interaction with the magnetic field. Because both the Li ground $2 \mathrm{~S}$ and first excited state $2 \mathrm{P}$ are energetically isolated from each other, and from all other excited levels, the motional stark effect is negligible and the Zeeman effect dominates the splitting and polarization of this transition. This transition is split into three components, usually labeled $\sigma$ and $\pi$. The $\pi$ component has a transition energy that is nominally independent of the magnetic field strength and is separated from the sigma lines by $>15 \mathrm{GHz}$ in TEXT. Since the $\pi$ line is polarized parallel to the local B field direction (when viewing perpendicular to B), laser excitation can be used to determine the local direction of $\mathrm{B}$. 


\section{DIAGNOSTIC SYSTEM}

The system is shown schematically in Fig. 1 . The Li beam consists of a Li ion gun, acceleration and iucusing optics, a steering magnet, and a Na vapor neutralization cell. Typically, the ion source is operated at about $4 \mathrm{~mA}$ emission, producing an ion beam current of $2.5 \mathrm{~mA}$ at $95 \mathrm{keV}$. The neutralization efficiency is about $40 \%$, resulting in an equivalent neutral current of $1 \mathrm{~mA}$. The laser, a modified cw dye laser pumed by an $\mathrm{Ar}$ ion laser, uses an acousto-optic modulator as a cavity-dumper to produce a 9 ns pulse at repetition rates from 0 to $2 \mathrm{MHz}$. In the data shown below, a $1 \mathrm{MHz}$ repetition rate was used. Peak pulse powers of over $20 \mathrm{~W}$ have been obtained. The laser beam is passed through a $\mathrm{LiNbO}_{3}$ polarization rotator, which maintains the linear polarization state of the laser light, but rotates the plane of polarization at a frequency of $50 \mathrm{kHz}$. The laser is merged with the $\mathrm{Li}$ beam, and the two beams are directed collinearly into the TEXT plasma and onto a transmitted Li beam detector. As discussed previously, ${ }^{1}$ the polarization rotation results in a laser excitation of the Li atoms that is modulated at $100 \mathrm{kHz}$, and the phase of this $100 \mathrm{kHz}$ modulation is directly related to the pitch angle of the magnetic field at the point of excitation.

The transmitted beam detector consists of a thin carbon foil of about $20 \mu \mathrm{g} / \mathrm{cm}^{2}$ surface density and an ion collector plate. The incoming $95 \mathrm{keV} \mathrm{Li}$ atoms are stripped of an electron as they pass through the foil. The collected current is then amplified using a $100 \mathrm{MHz}$ bandwidth transimpedance amp located adjacent to the collector and the signal is conducted out of the vessel using $50 \Omega$ coax. The foil, about $2 \mathrm{~cm}$ in diameter, is supported by fine stainless steel mesh and is protected from the plasma by a $2 \mathrm{mr}$ uhick hexagonal collimator with $1 \mathrm{~mm}$ holes. The detector is insensitive to incoming photons (UV and X-ray) since secondary electrons are unable to escape from the collector due to the large ambient magnetic field. 


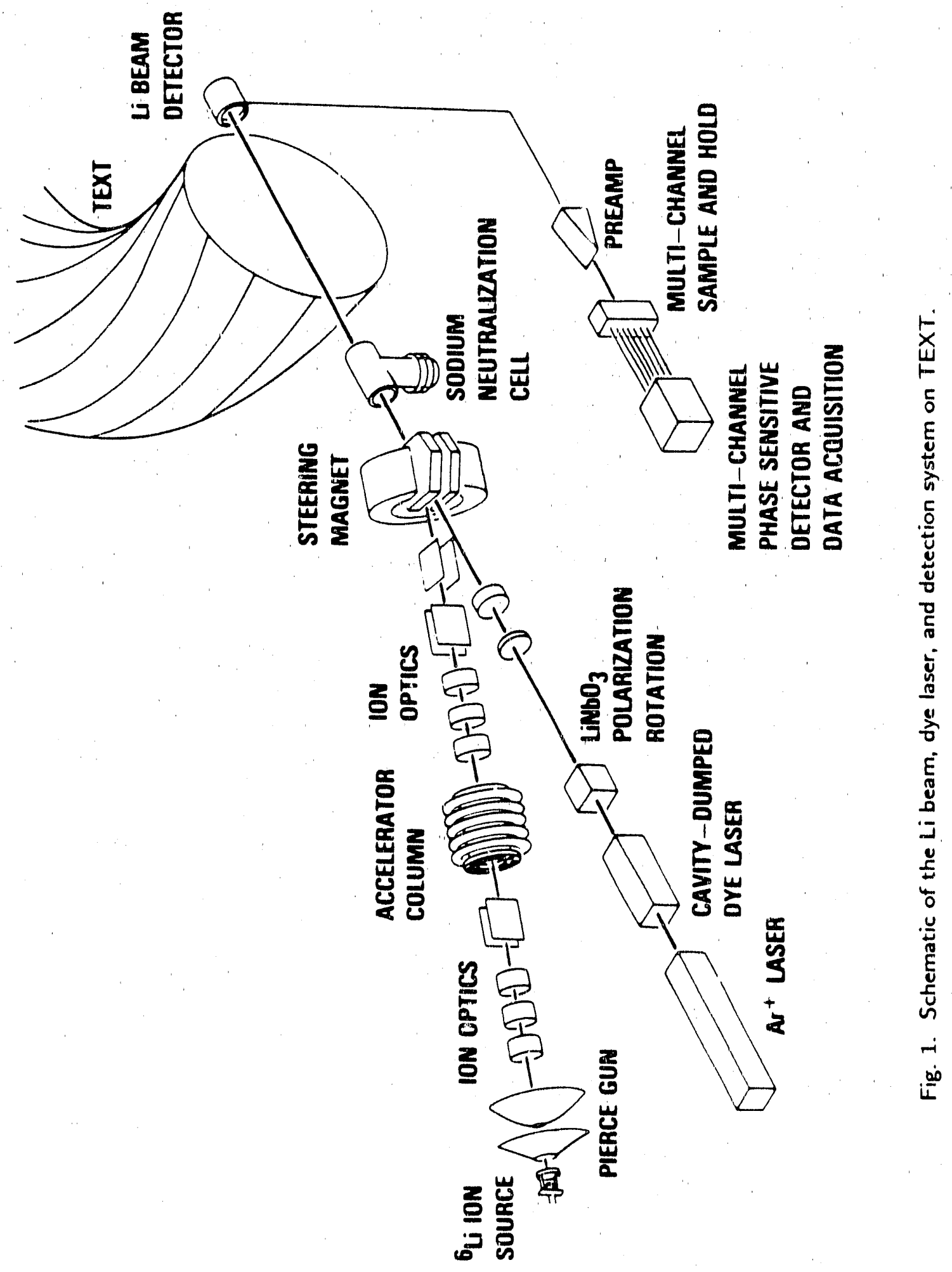


The signal, after secondary amplification, is directed to $100 \mathrm{MHz}$ bandwidth track and hold amps $(\mathrm{T} \& \mathrm{H})$. Each T\&H is triggered to hold the value of the signal at a precise delay time from the laser pulse. These delay times are related to the propagation time of the timing pulses, the signal, and the Li beam atoms from particular radial positions in the tokamak to the detector. The radial resolution of the measurement, determined by the pulse length of the laser, the bandwidth of the electronics, and the velocity of the beam atoms, is about $2 \mathrm{~cm}$. After the T\&H's, each signal is directed to a phasesensitive detector, locked to the polarization rotator, to measure the relative phase of the effect of the polarization rotation on the $\mathrm{Li}$ beam at a localized position in the plasma. At present, we have operaten with only one T\&H.

\section{EXPERIMENTAL DATA}

Pitch angle measurements obtained using this system are shown in Fig. 2 along with a few relevant TEXT plasma parameters. These data have been averaged over $10 \mathrm{~ms}$ and over 19 similar shots. The pitch at $r=+15 \mathrm{~cm}$ clearly follows the plasma current, as is expected. The observed change in the measured pitch during the initial plasma current ramp from 0 to $50 \mathrm{~ms}$ is reasonable for this radial position and plasma current. The pitch at $r=-4 \mathrm{~cm}$ should not be correlated with the plasma current, since it is very near the magnetic axis.

Absolute pitch of $B$ was not determined from this data because there was no LEA effect prior to the discharge initiation. In previous work using LIF, there was a strong signal just prior to the discharge allowing determination of absolute pitch. Due to lack of cross section information, we were unable to predict the size of LEA in the neutral hydrogen gas injected $50 \mathrm{~ms}$ prior to the discharge, but this data indicates that it is 


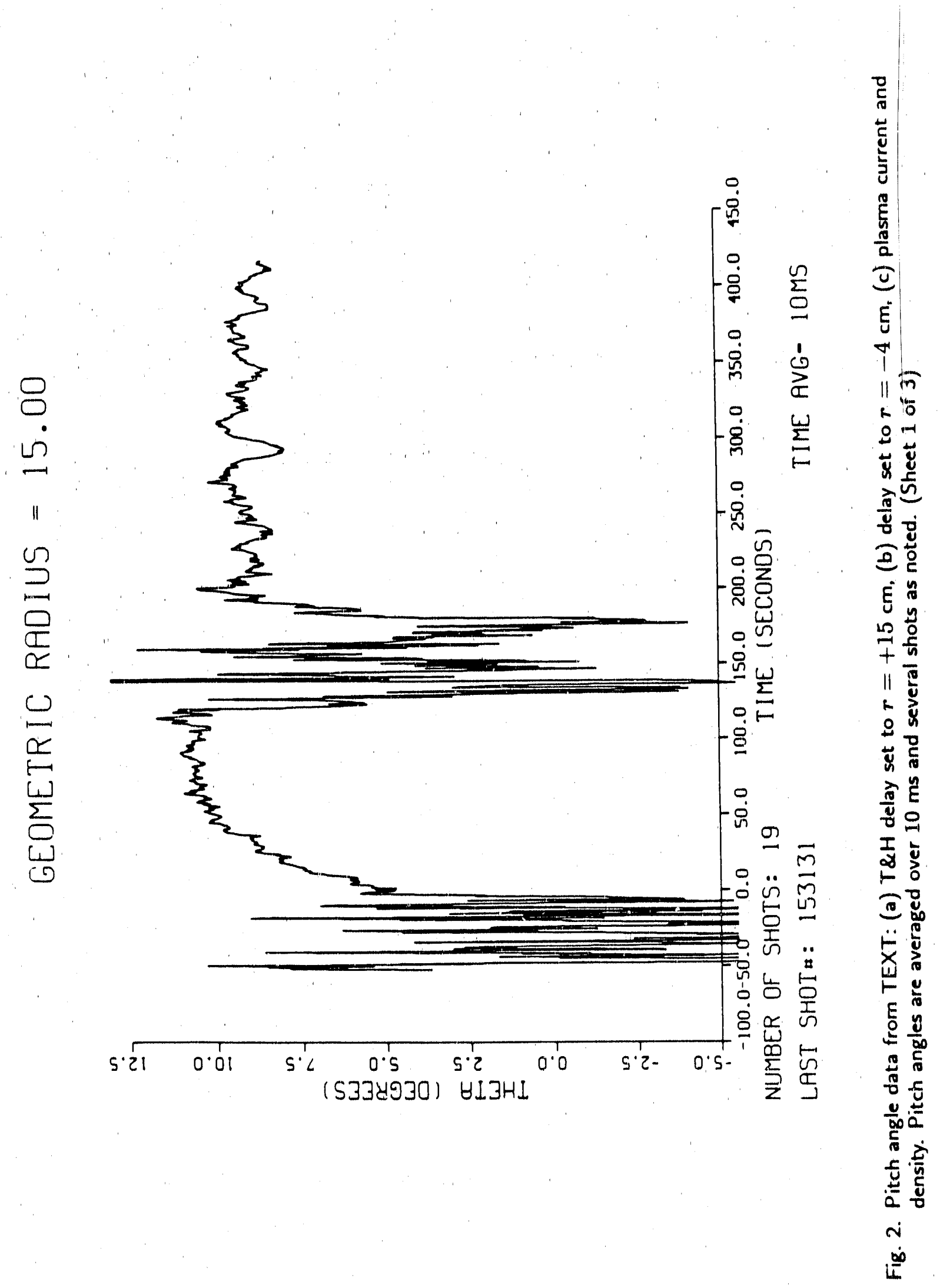




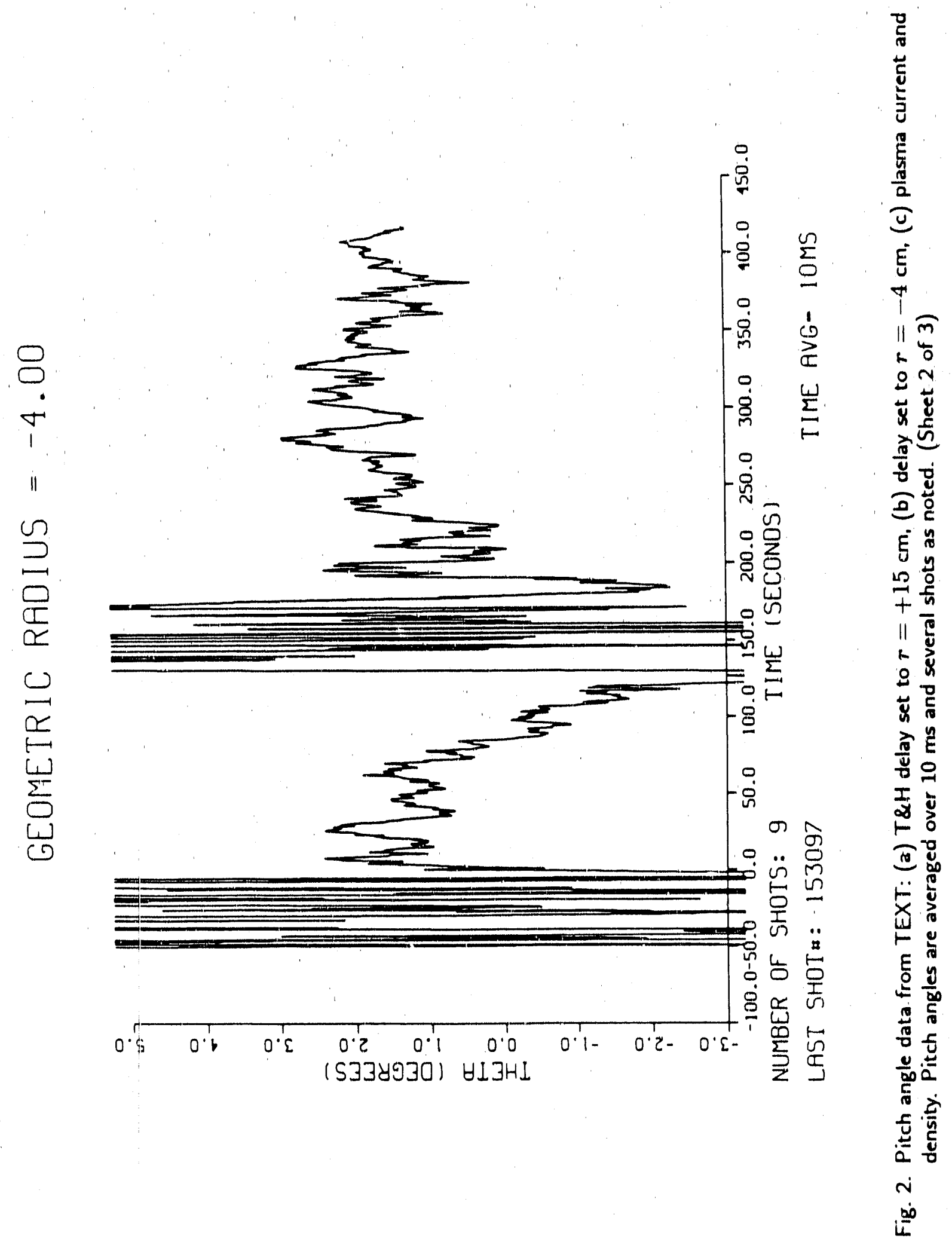


Fir Dens Centrl $\left(10^{13} \mathrm{~cm}^{-3}\right)$

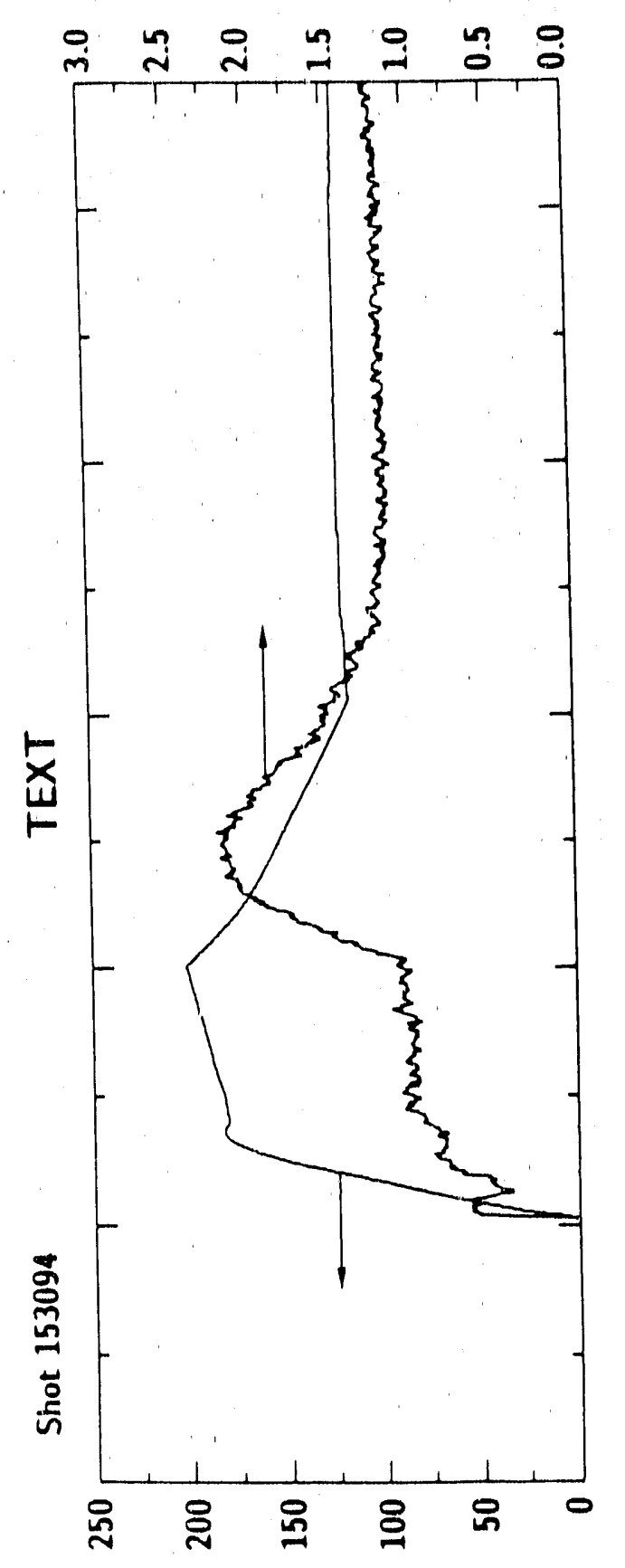

$(\forall y)$ juadun euseld

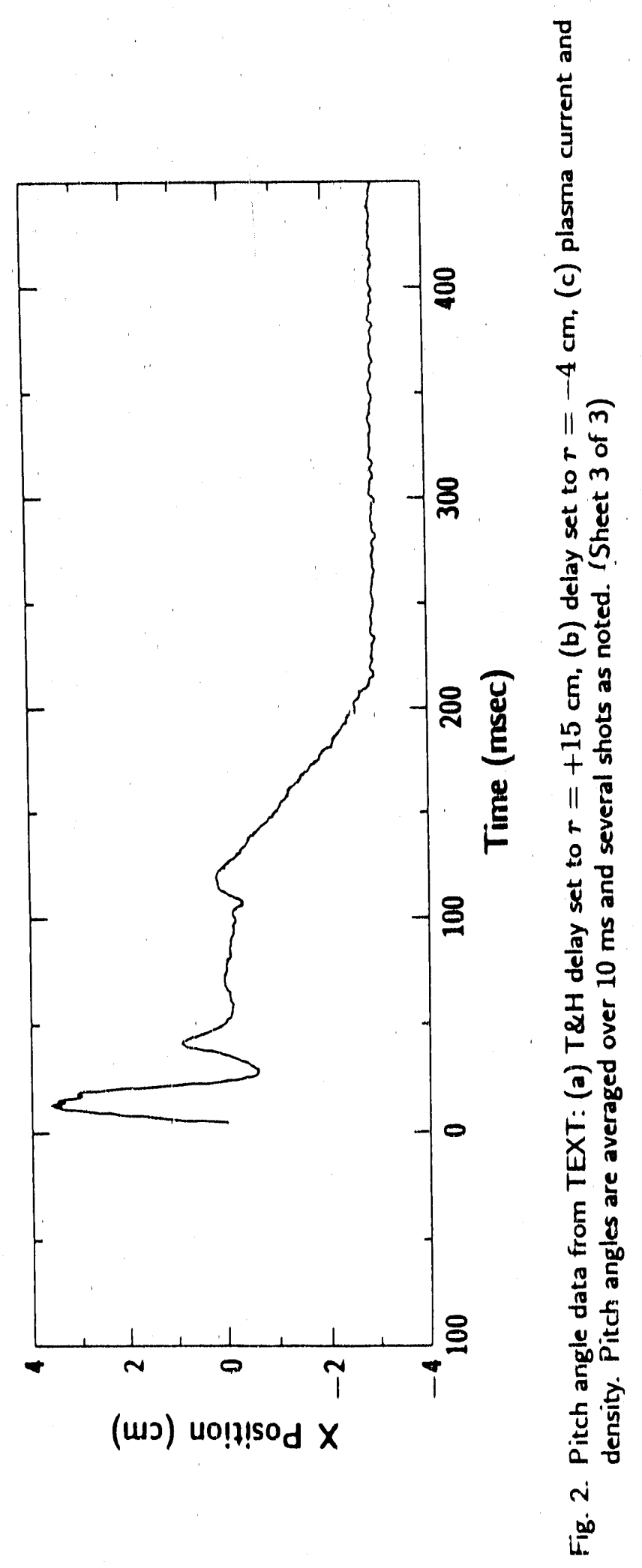


quite small. As discussed below; we are developing a optical reference that will allow absolute pitch determination.

The signal-to-noise in this data was well below that initially expected due to two reasons: (1) the laser pulse peak power was only $5 \mathrm{~W}$, significantly below the $20 \mathrm{~W}$ achieved in the past, (2) the major contributor to the observed noise was pickup from plasma fluctuations. As a result, as the plasma density was ramped above $1.5 \times$ $10^{13} \mathrm{~cm}^{-3}$ (between 100 and $200 \mathrm{~ms}$ ), the transmitted Li beam is too weak to observe the LEA effect.

Shown in Fig. 3 is an FFT power spectrum obtained by digitizing the output of the $\mathrm{T} \& \mathrm{H}$ at $500 \mathrm{kHz}$ sample rate. The signal at $100 \mathrm{kHz}$ is clearly observable, as well as artifacts at 50 and $150 \mathrm{kHz}$ due too poor alignment of the polarization rotator. Also observable is a band of noise centered near $100 \mathrm{kHz}$ that we attribute to pickup of edge plasma turbulence.

\section{DISCUSSION}

Several improvernents to the system are suggested by the data presented here. First, to improve the signal-to-noise, the laser must be brought back to previous performance levels and the pulse rate can be doubled to $2 \mathrm{MHz}$. In addition, the effect of plasria turbulence can be reduced by improving the shielding of the detector and by moving the frequency of laser rotation to above $130 \mathrm{kHz}$. We expect an improvement in signal-to-noise by more than an order of magnitude. Increasing the $\mathrm{Li}$ beam current will also improve $\mathrm{S} / \mathrm{N}$, but is not a trivial change.

A phase reference suitable for determining absolute pitch has been designed. Directing the small percentage of the laser beam that is reflected from the vacuum input 


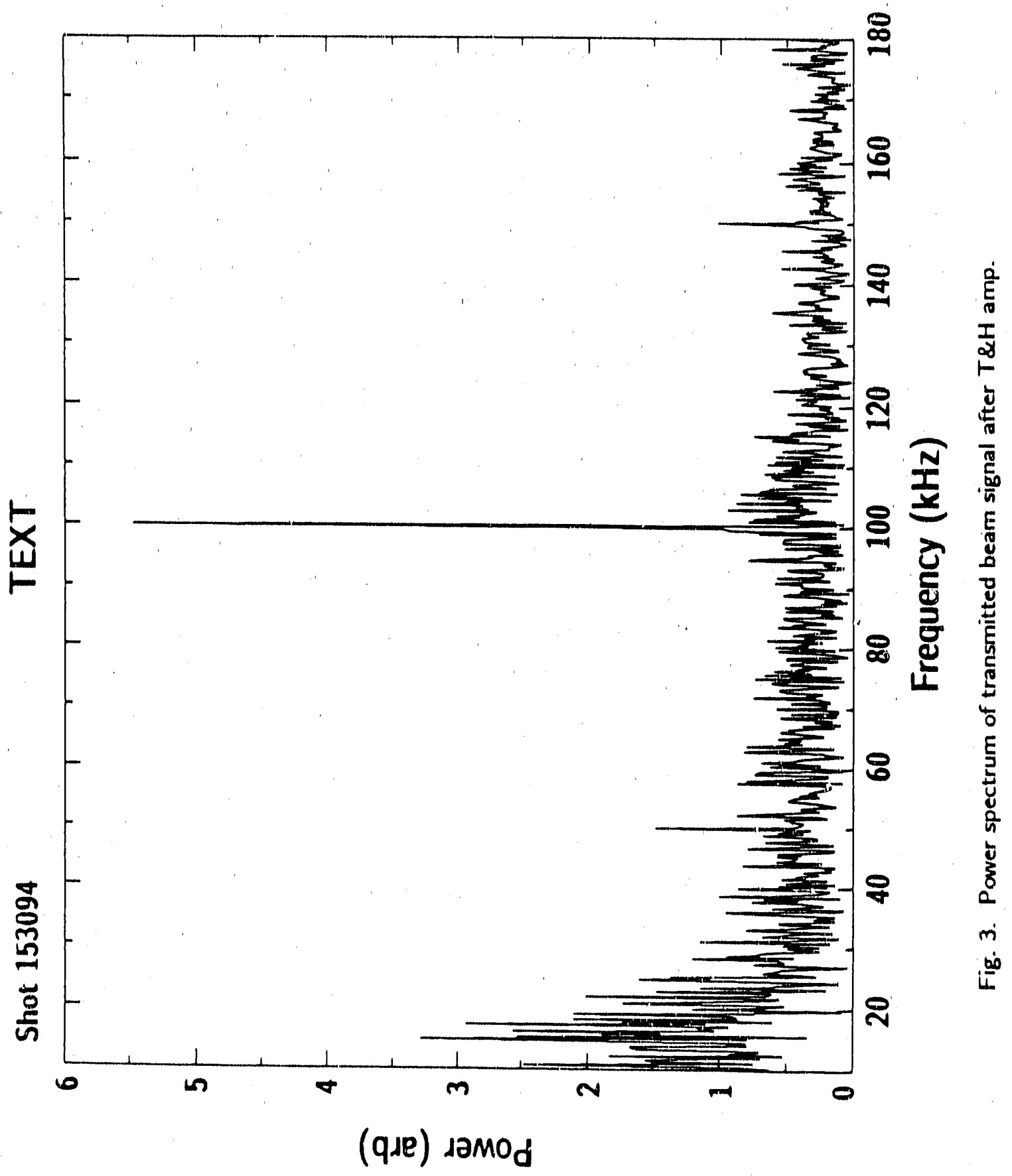


window through a polarization beam splitting cube and onto a plotodiode yields a signal that is directly related to the orientation of the cube. The cube is accurately aligned to the local horizontal using a precision spirit level. The signal is then directed to a $\mathrm{T} \& \mathrm{H}$ amp identical to those used for the transmitted beam signal. In order to avoid phase shifts usually found in analog electronics, the phase-sensitive detectors will be removed, and the signal from the $\mathrm{T} \& \mathrm{H}$ arnps will be directly digitized in sync with their triggers. The phase comparison between the photodiode signal and the transmitted beam signals will be done digitally.

In conclusion, the initial magnetic pitch data obtained on TEXT using the LEA technique is very promising. We anticipate that following the shutdown for TEXT upgrade, we can implement the improvements discussed above and obtain complete radial profiles with good time resolution.

\section{ACKNOWLEDGMENT}

This work was supported by the U.S. Department of Energy under Contract No. DE-AC03-89ER53277. 


\section{REFERENCES}

1 W. P. West, D. M. Thomas, E. S. Ensberg, J. S. deGrassie, and J. F. Baur, Rev. Sci. Instrum. 57, 1552 (1986).

2 W. P. West, D. M. Thomas, J. S. deGrassie, and S. B. Zheng, Phys. Rev. Lett. 58, 2758 (1987).

3 W. P. West, D. M. Thomas, and M. P. Thomas, Rev. Sci. Instrum. 59, 1617 (1988). 

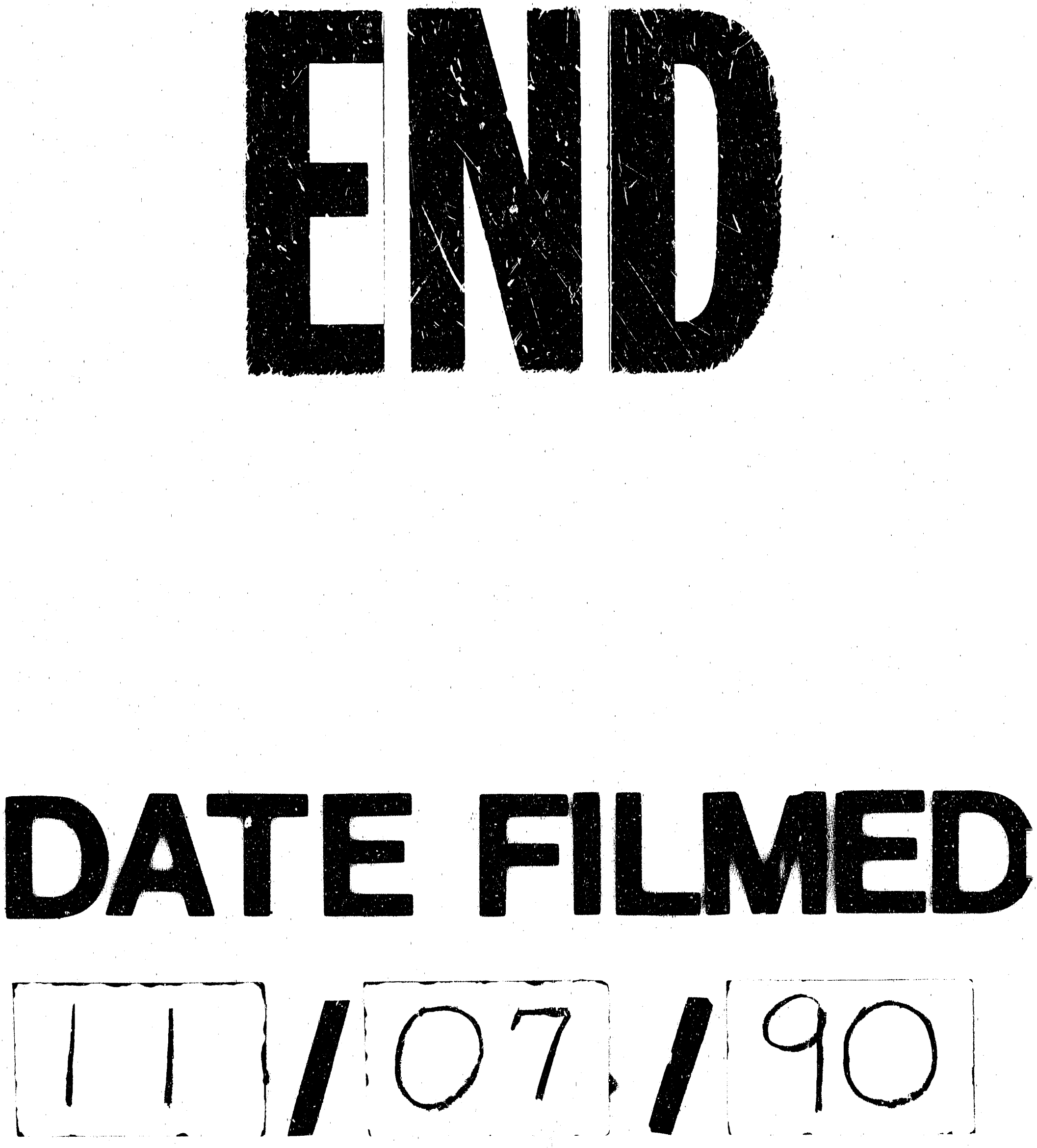
